\title{
Clinical determinants of long-term survival in metastatic uveal melanoma
}

\author{
Elias A. T. Koch ${ }^{1,2} \cdot$ Anne Petzold $^{1,2} \cdot$ Anja Wessely $^{1,2} \cdot$ Edgar Dippel $^{3} \cdot$ Michael Erdmann $^{1,2} \cdot$ Lucie Heinzerling $^{1,4}$. \\ Bettina Hohberger ${ }^{5} \cdot$ Harald Knorr $^{5}$. Ulrike Leiter $^{6} \cdot$ Friedegund Meier $^{7} \cdot$ Peter Mohr $^{8} \cdot$ Farnaz Rahimi $^{4}$. \\ Beatrice Schell ${ }^{9} \cdot$ Max Schlaak $^{4,10} \cdot$ Patrick Terheyden $^{11} \cdot$ Beatrice Schuler-Thurner $^{1,2} \cdot$ Selma Ugurel ${ }^{12}$. \\ Jochen Utikal $^{13,14}$. Julio Vera ${ }^{1,2} \cdot$ Michael Weichenthal $^{15} \cdot$ Fabian Ziller $^{16} \cdot$ Carola Berking $^{1,2} \cdot$ Markus V. Heppt $^{1,2} \mathbb{D}$
}

Received: 2 June 2021 / Accepted: 12 October 2021 / Published online: 28 October 2021

(c) The Author(s) 2021

\begin{abstract}
This study aimed to identify prognostic factors in patients with metastatic uveal melanoma (UM) that were associated with long-term survival in a real-world setting. A total of 94 patients with metastatic UM were included from German skin cancer centers and the German national skin cancer registry (ADOReg). Data were analyzed for the response to treatment, progression-free survival, and overall survival (OS). Prognostic factors were explored with univariate Cox regression, $\log$-rank, and $\chi 2$-tests. Identified factors were subsequently validated after the population was divided into two cohorts of short-term survival ( $<2$ years OS, cohort A, $n=50$ ) and long-term survival ( $>2$ years OS, cohort $\mathrm{B}, n=44$ ). A poor ECOG performance status (hazard ratio [HR] 2.0, 95\% confidence interval [CI] 1.0-3.9) and elevated serum LDH (HR 2.0, 95\% CI 1.0-3.8) were associated with a poor OS, whereas a good response to immune checkpoint blockade (ICB, $p<0.001$ ), radiation therapy $(p<0.001)$, or liver-directed treatments $(p=0.01)$ were associated with a prolonged OS. Long-term survivors (cohort B) showed a higher median number of organs affected by metastasis $(p<0.001)$, while patients with liver metastases only were more common in cohort A ( $40 \%$ vs. $9 \% ; p=0.002)$. A partial response to ICB was observed in $16 \%(12 / 73)$, being $21 \%$ (8/38) for combined ICB, 17\% (1/6) for single CTLA4 inhibition, and 10\% (3/29) for single PD1 inhibition. One complete response occurred in cohort B with combined ICB. We conclude that the response to ICB and the presence of extrahepatic disease were favorable prognostic factors for long-term survival.
\end{abstract}

Keywords Uveal melanoma $\cdot$ Immune checkpoint blockade $\cdot$ Liver metastases $\cdot$ Liver-directed treatment $\cdot$ Long-term survival · Registry

\section{Introduction}

Uveal melanoma (UM) is the most common tumor of the eye in adults but still represents a rare subtype of melanoma. The mean age-adjusted incidence is 5.2 per million [1] and shows

Précis Data from 94 patients with metastatic uveal melanoma were analyzed to identify prognostic factors that were associated with long-term survival in a real-world setting. In particular, achieving a response to treatment with immune checkpoint blockade and, paradoxically, the presence of multiple metastatic sites with extrahepatic metastasis were favorable prognostic factors.

Markus V. Heppt

markus.heppt@uk-erlangen.de

Extended author information available on the last page of the article a north-to-south decreasing gradient in Europe [2], with the highest current mean incidence of 9.5 per million in Ireland [3]. The treatment approach of the primary tumor depends on the tumor size, patient preference, and tumor localization, most commonly by brachytherapy or enucleation $[4,5]$. The most important risk factor for the development of metastases is monosomy 3 , and at least $40-50 \%$ of patients will develop metastases, predominantly to the liver $[6,7]$. Since there is no standardized and effective treatment for advanced $\mathrm{UM}$, the prognosis is bleak once metastases develop [8]. A meta-analysis of studies published between 1980 and 2017 including 2494 patients calculated a median overall survival (OS) across all treatment modalities of 1.07 years [9]. However, the population of this meta-analysis was treated mainly in the time before immune checkpoint blockade (ICB) was available. In both settings, some patients show 
a more favorable disease course with longer OS. Thus, this study aimed to identify parameters that are linked to a more favorable clinical outcome in patients with metastatic UM.

\section{Patients and methods}

\section{Patient population and data sources}

We performed a retrospective, multi-center, explorative analysis. Patients with metastatic UM receiving any treatment regime were eligible. Inclusion criteria were histologically confirmed stage IV UM and a follow-up time of at least three months. As we were interested in determinants of longterm survival, the following exclusion criteria applied: (1) unknown survival status within two years after diagnosis of stage IV disease, (2) unknown date of entry into stage IV, and (3) ongoing treatment and a survival time of fewer than two years at the data cut-off. These criteria were set as it was unclear at the time of the data cut-off if patients under these conditions would, later on, turn out as long-term survivors. Clinical data and the treatment outcomes of interest were extracted from the original patient records from three German skin cancer centers (Universitätsklinikum Erlangen $n=43$, University Hospital Munich $n=3$, and Klinikum Ludwigshafen $n=2$ ), as well as from the ADOREG registry of the German Dermatologic Cooperative Oncology Group (DeCOG, $n=46$ ). Data were collected and merged into a central database before analysis. The ADOReg registry is a large prospective clinical database in the field of dermatologic oncology collecting data to generate high-quality realworld evidence. This study was approved by the scientific board of the registry and by the institutional review board of the medical faculty of the Munich University Hospital (approval number 413-16 UE). Furthermore, it was conducted following the principles of the Helsinki Declaration in its current version.

\section{Data collection and treatment outcomes}

The clinical data recorded at the diagnosis of stage IV UM ("baseline") comprised demographics such as sex, age, number, and sites of metastases, the Eastern Cooperative Oncology Group (ECOG) performance status, and serum lactate dehydrogenase $(\mathrm{LDH})$ as a dichotomous variable (elevated vs. not elevated; cut-off $250 \mathrm{U} / \mathrm{l}$ ). The most common metastatic sites such as liver, bone, lung, and central nervous system (CNS) were specified, while all other localizations were summarized within the category "other metastases." No further information was available on the extent of hepatic metastasis.

We recorded the number and types of treatments and further dissected ICB regarding start and end date, time to progression, and best radiologic response evaluation based on the RECIST criteria version 1.1. The best radiologic response to ICB treatment which was achieved during the disease course was assessed by the site investigators and indicated as complete response (CR), partial response (PR), stable disease (SD), or progressive disease (PD). In all cases, patients were treated until disease progression or development of unacceptable toxicity. As the treatments were highly heterogeneous, we subsumed interventions other than ICB, cytotoxic chemotherapy, and vaccination with dendritic cells loaded with tumor-intrinsic RNA (DC) as a category "other treatments." Besides, we extracted data on the performance of liver-directed treatments and radiation therapy.

\section{Statistical analysis}

OS was calculated as the time from the diagnosis of stage IV UM until melanoma-specific or treatment-related death. The progression-free survival (PFS) was determined as the time from treatment start until disease progression. Time-toevent analyses were calculated where death or progression was considered as an event. If neither occurred or if patients were lost to follow-up, the date of the last documented presentation was used as a censored observation.

The survival and progression probabilities were estimated with the Kaplan-Meier method. To test for a significant moderating factor, the survival curves were compared with the log-rank test. The hazard ratio (HR) with $95 \%$ confidence interval (CI) was calculated by the COX proportional hazard regression whenever the ability for assuming the proportional hazards was given (no crossing of survival curves in the $\log -\log$ plot). In this case, the $p$-values were calculated with the Wald test. If the proportional hazard assumption was violated, only the different median OS was indicated. Patients with missing values for a given variable were excluded. No imputation of missing data was performed.

For investigating possible factors being significantly different in the groups of short-time survivors (cohort A) vs. long-time survivors (cohort B), log-rank tests, $\chi^{2}$-tests and $\mathrm{t}$-tests were performed. In all cases, two-tailed $\mathrm{p}$-values were calculated and considered significant with values $p<0.05$. All analyses were carried out with the software R (https:// www.r-project.org/) using the packages "survival" and "survminer."

\section{Results}

\section{Characteristics of the study population}

A total of $94(100 \%)$ patients with metastatic UM met the eligibility criteria and were included (Table 1). A median of three organ systems was affected by metastases, 
Table 1 Characteristics of the study population

\begin{tabular}{|c|c|c|c|c|c|}
\hline Parameter & Categories & Number $(\%) N=94(100 \%)$ & Group A $N=50$ & Group B $N=44$ & $p$-value \\
\hline Age & Median in years & 67 (range 33-92) & 67.4 & 65.8 & $p=0.85$ \\
\hline \multirow[t]{2}{*}{ Sex } & Female & $41(44 \%)$ & $22(44 \%)$ & $19(43 \%)$ & \multirow[t]{2}{*}{$p=1$} \\
\hline & Male & $53(55 \%)$ & $28(56 \%)$ & $25(57 \%)$ & \\
\hline \multirow[t]{6}{*}{ ECOG performance status } & 0 & $39(42 \%)$ & $17(34 \%)$ & $22(50 \%)$ & \multirow[t]{6}{*}{$p=0.17$} \\
\hline & 1 & $12(13 \%)$ & $5(10 \%)$ & $7(16 \%)$ & \\
\hline & 2 & $3(3 \%)$ & $3(6 \%)$ & $0(0 \%)$ & \\
\hline & 3 & $1(1 \%)$ & $1(2 \%)$ & $0(0 \%)$ & \\
\hline & 4 & $0(0 \%)$ & $0(0.0 \%)$ & $0(0.0 \%)$ & \\
\hline & Not indicated & $39(42 \%)$ & $24(48 \%)$ & $15(34 \%)$ & \\
\hline \multirow[t]{3}{*}{ LDH } & Not elevated & $22(23 \%)$ & $9(18 \%)$ & $13(30 \%)$ & \multirow[t]{3}{*}{$p=0.02$} \\
\hline & Elevated & $36(38 \%)$ & $27(54 \%)$ & $9(20 \%)$ & \\
\hline & Not indicated & $36(38 \%)$ & $14(28 \%)$ & $22(50 \%)$ & \\
\hline \multirow[t]{9}{*}{ Sites of metastasis } & Liver & $87(93 \%)$ & $48(96 \%)$ & $39(89 \%)$ & $p=0.3$ \\
\hline & Hepatic only & $24(26 \%)$ & $20(40 \%)$ & $4(9 \%)$ & $p=0.001$ \\
\hline & Both hepatic and extrahepatic & $63(67 \%)$ & $28(56 \%)$ & $35(80 \%)$ & $p=0.028$ \\
\hline & Extrahepatic only & $7(7 \%)$ & $2(4 \%)$ & $5(11 \%)$ & $p=0.34$ \\
\hline & Pulmonary & $48(51 \%)$ & $20(40 \%)$ & $28(64 \%)$ & $p=0.04$ \\
\hline & Bone & $32(34 \%)$ & $16(32 \%)$ & $16(36 \%)$ & $p=0.8$ \\
\hline & CNS & $20(21 \%)$ & $7(14 \%)$ & $13(30 \%)$ & $\mathrm{p}=0.1$ \\
\hline & Other sites & $42(45 \%)$ & $13(26 \%)$ & $29(66 \%)$ & $p<0.001$ \\
\hline & Not indicated & $2(2 \%)$ & $0(0 \%)$ & $2(5 \%)$ & $p=0.42$ \\
\hline Number of metastatic sites & Median (range) & $3(1-5)$ & $2(1-5)$ & $3(1-5)$ & $p<0.001$ \\
\hline \multirow[t]{8}{*}{ Systemic treatments } & Vaccination with dendritic cells & $6(6.4 \%)$ & $1(2.0 \%)$ & $5(11.4 \%)$ & $p=0.15$ \\
\hline & Any immune checkpoint blockade & $81(86 \%)$ & $41(82 \%)$ & $40(91 \%)$ & $p=0.15$ \\
\hline & Single CTLA4 inhibition & $8(9 \%)$ & $2(4 \%)$ & $6(14 \%)$ & $p=0.19$ \\
\hline & Single PD-1 inhibition & $33(35 \%)$ & $17(34 \%)$ & $16(38 \%)$ & $p=0.98$ \\
\hline & $\begin{array}{l}\text { Combined immune checkpoint block- } \\
\text { ade }\end{array}$ & $40(43 \%)$ & $22(44 \%)$ & $18(41 \%)$ & $p=0.93$ \\
\hline & Not indicated & $2(2 \%)$ & $1(2 \%)$ & $1(2 \%)$ & $p=1$ \\
\hline & Chemotherapy & $29(31 \%)$ & $9(18 \%)$ & $20(46 \%)$ & $p=0.02$ \\
\hline & $\begin{array}{l}\text { Reinduction with immune checkpoint } \\
\text { blockade }\end{array}$ & $33(35 \%)$ & $9(18 \%)$ & $24(55 \%)$ & $p=0.001$ \\
\hline \multirow[t]{3}{*}{ Liver-directed treatment } & Yes & $35(37 \%)$ & $17(34 \%)$ & $18(41 \%)$ & \multirow[t]{3}{*}{$p=0.01$} \\
\hline & No & $41(44 \%)$ & $32(64 \%)$ & $9(21 \%)$ & \\
\hline & Unknown & $18(19 \%)$ & $1(2 \%)$ & $17(39 \%)$ & \\
\hline \multirow[t]{3}{*}{ Radiation therapy } & Yes & $23(25 \%)$ & $8(16 \%)$ & $15(34 \%)$ & \multirow[t]{3}{*}{$p=0.001$} \\
\hline & No & $53(56 \%)$ & $42(84 \%)$ & $9(21 \%)$ & \\
\hline & Unknown & $18(19 \%)$ & $0(0 . \%)$ & $18(41 \%)$ & \\
\hline
\end{tabular}

predominantly liver (93\%), lung (51\%), and bones (34\%); $42 \%$ had an ECOG performance status of 0 . Serum LDH was elevated in $38 \%$ when stage IV disease was entered.

The median OS of the entire population was 22.3 months (95\% CI 14.9-26.7), the median PFS in stage IV disease after the first systemic treatment was 3.0 months (95\% CI 2.4-3.7) (Fig. 1A + B). The majority of patients received any ICB (86\%), while liver-directed treatments and radiation therapy were applied in $37 \%$ and
$25 \%$, respectively. One CR occurred in a patient undergoing combined ICB $(1 \%, 1 / 73)$. The PR rate for all ICB regimens was $16 \%(12 / 73)$, being $21 \%$ for combined ICB (8/38), $17 \%$ for single CTLA4 inhibition (1/6), and $10 \%$ for single PD1 inhibition (3/29, Table 2). The median PFS after ICB was 2.5 months (95\% CI 2.1-3.5). In cohort B, the rate of PR was $14 \%(2 / 14)$ and $41 \%$ (7/17) for single PD1 inhibition and combined ICB, respectively. One CR $(6 \%, 1 / 17)$ occurred in this cohort (Table 3$)$. 

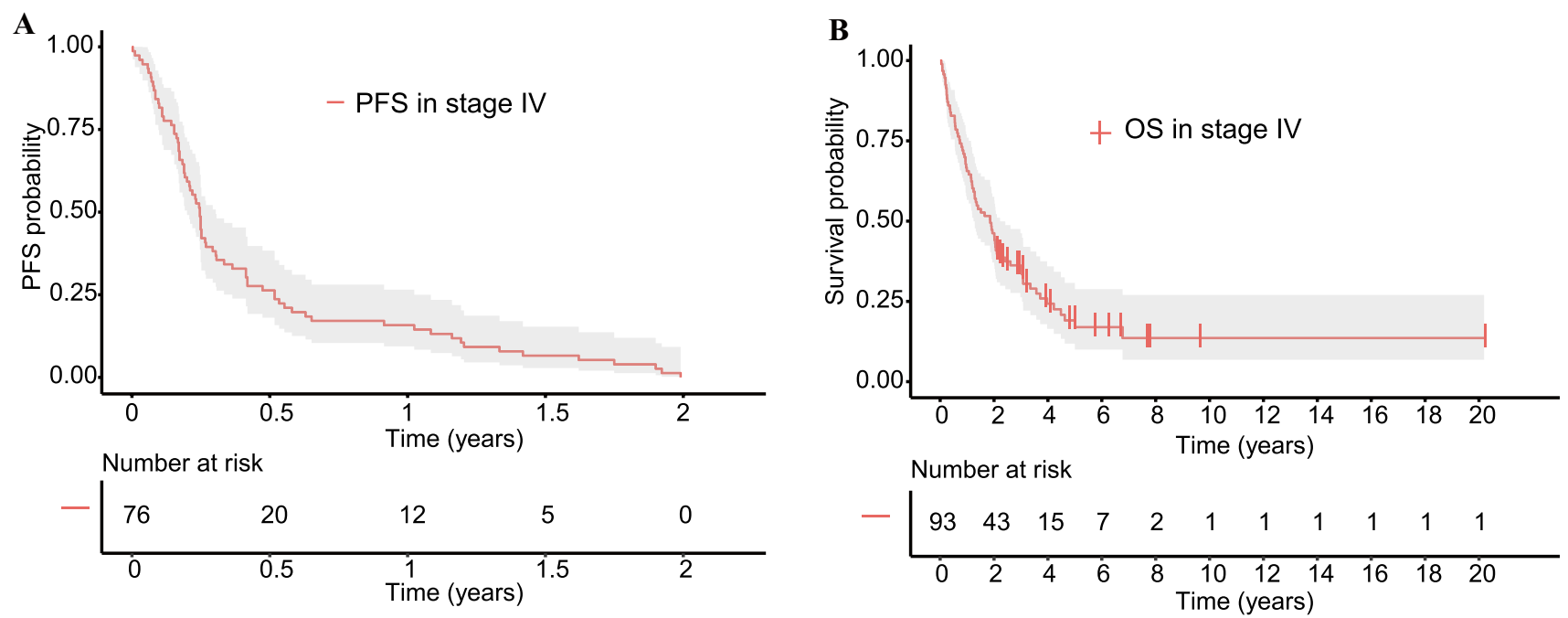

Fig. 1 Kaplan-Meier estimates of the patient population for (A) progression-free survival (PFS) to first systemic therapy and (B) overall survival (OS). The median PFS and OS were 3.0 months (95\% CI 2.4-3.7) and 22.3 months (95\% CI 14.9-26.7), respectively

Table 2 Partial response rates of short-term (cohort A) versus long-term (cohort B) survivors

\begin{tabular}{lllll}
\hline ICB regimen & Total $(n)$ & Cohort A $(n)$ & Cohort B $(n)$ & $p$-value \\
\hline Any ICB & $16 \%(12 / 73)$ & $5 \%(2 / 38)$ & $29 \%(10 / 35)$ & $p=0.018$ \\
Single PD1 inhibition & $10 \%(3 / 29)$ & $7 \%(1 / 15)$ & $14 \%(2 / 14)$ & $p=0.95$ \\
Single CTLA4 inhibition & $17 \%(1 / 6)$ & $0 \%(0 / 2)$ & $25 \%(1 / 4)$ & $p=1$ \\
Combined ICB & $21 \%(8 / 38)$ & $5 \%(1 / 21)$ & $41 \%(7 / 17)$ & $p=0.019$ \\
\hline
\end{tabular}

ICB immune checkpoint blockade

\begin{tabular}{llcc}
\hline $\begin{array}{l}\text { ICB regimen } \\
\text { cohort B) }\end{array}$ & $\begin{array}{l}\text { Complete } \\
\text { response }\end{array}$ & Partial response & $\begin{array}{l}\text { No response (stable dis- } \\
\text { ease, progressive disease) }\end{array}$ \\
\hline Any ICB $(n=35)$ & $1(3 \%)$ & $10(29 \%)$ & $24(69 \%)$ \\
Single PD1 inhibition $(n=14)$ & $0(0 \%)$ & $2(14 \%)$ & $12(86 \%)$ \\
Single CTLA4 inhibition $(n=4)$ & $0(0 \%)$ & $1(25 \%)$ & $3(75 \%)$ \\
Combined ICB $(n=17)$ & $1(6 \%)$ & $7(41 \%)$ & $9(53 \%)$ \\
\hline
\end{tabular}

ICB immune checkpoint blockade

\section{Identification of prognostic factors}

We next performed univariate analysis on the entire population to identify putative prognostic factors. Neither sex nor age was associated with OS (Fig. 2A+B). Both a poor ECOG performance status (HR 2.0, 95\% CI 1.0-3.9) and elevated serum LDH level (>250 U/l; HR $2.0,95 \%$ CI 1.0-3.8) were unfavorable prognostic factors (Fig. 2C+D). The site and number of metastases were not associated with OS except for the category "other metastases" ( $p=0.009)$ (Suppl. Figure 1, Suppl. Table 1). Patients with liver metastases only showed significantly worse survival compared to those with a combination of both hepatic and extrahepatic metastases (median OS 7.7 vs. 24.8 months, respectively; $p=0.019$; Suppl. Figure $1 \mathrm{G}$ ).

Regarding treatments, the univariate analysis demonstrated prolonged survival of patients with three or more treatment lines $(p=0.002)$, "other treatments" $(p=0.054)$, and DC vaccination although the absolute numbers were low (HR 0.24, 95\% CI 0.06-0.96; Fig. 3A-C, Suppl. Table 4). Also, patients undergoing radiation therapy (HR 0.33, 95\% CI 0.18-0.60) or liver-directed treatments (HR 0.44, 95\% CI 0.26-0.76) showed more favorable OS (Fig. 3D +E, Suppl. Table 4). The best response to ICB was a strong prognostic factor $(p<0.001 ;$ Fig. $3 \mathrm{H})$, as was the reinduction of ICB ( $p=0.001$; Fig. 3I, Suppl. Table 4$)$. There was no significant difference in OS between single 

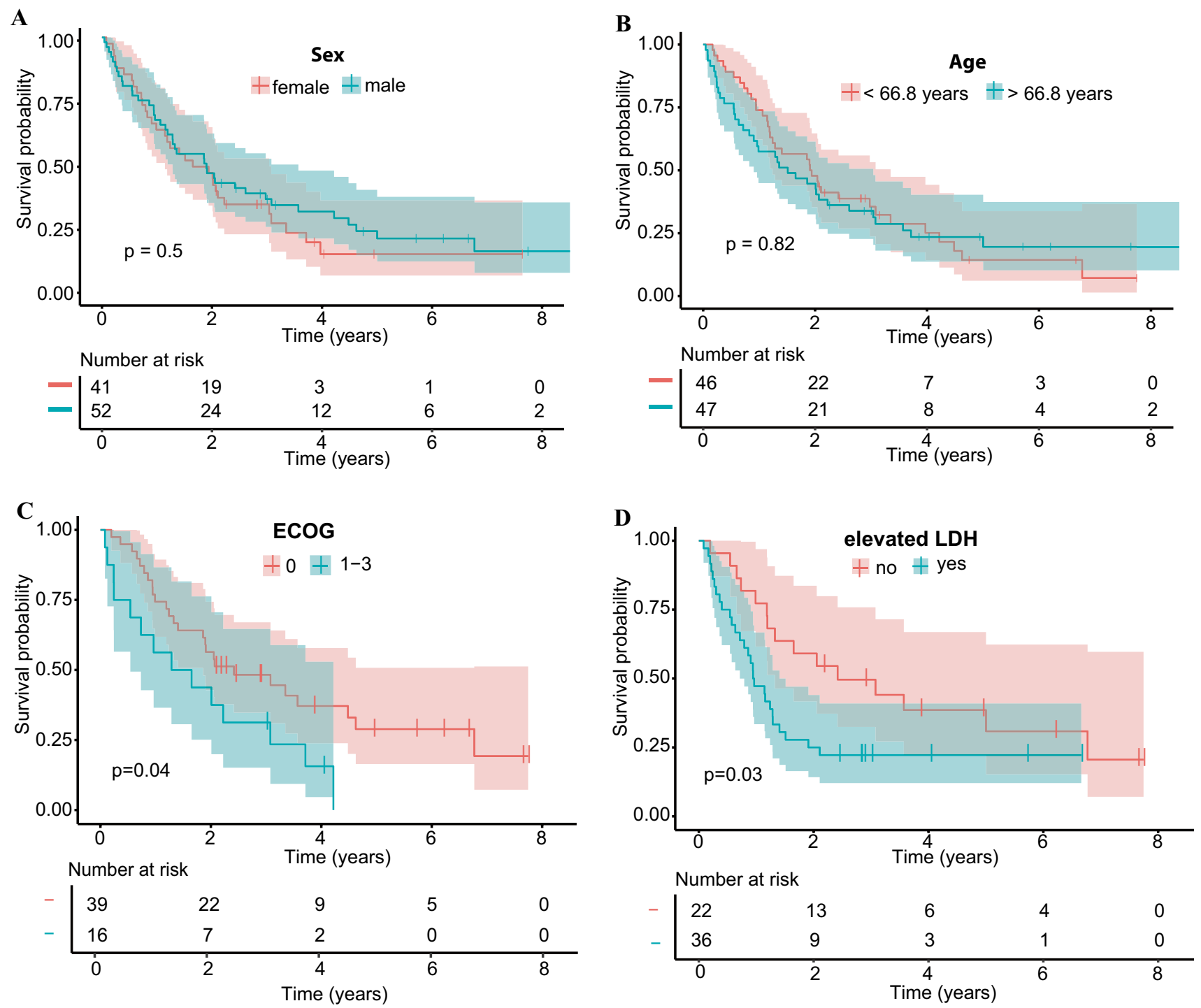

Fig. 2 A Kaplan-Meier estimates for overall survival (OS) according to sex. The median OS was 19.7 (95\% CI 13.8-36.4) for females vs. 22.6 months (95\% CI 14.2-37.4) for males. B OS according to age. The median OS was 23 (95\% CI 14.8-40.1) for $<66.8$ years vs.

PD1 and combined ICB ( $p=0.79$; Fig. 3G, Table Suppl. Table 4).

\section{Comparison of short-term and long-term survivors}

To further validate prognostic factors regarding long-term OS, the study population was subsequently divided into two subgroups: (1) cohort A $(n=50)$ with patients who died within the first two years after they entered stage IV disease and (2) cohort B $(n=44)$ consisting of patients with a survival of longer than 2 years. Age, sex, and ECOG performance status were evenly distributed among both cohorts (Fig. 4A). In contrast, elevated serum LDH levels were more commonly observed in cohort A $(p=0.02)$. Interestingly,

18.2 months (95\% CI 10.9-36.4) for $>66.8$ years. C OS according to ECOG performance status $(\mathrm{HR} \approx 2, p=0.04)$. D OS according to serum $\mathrm{LDH}$ level $(\mathrm{HR} \approx 2, p=0.03)$

patients in cohort B had a higher median number of affected organ systems $(p<0.001)$, including more patients with lung $(p=0.04)$ and "other metastases" $(p<0.01)$, while liver, bone, and CNS metastases were evenly distributed (Fig. 4B). Patients with liver metastases only were more common in cohort A than in B (40\% vs. $9 \%$; $p=0.002)$.

Both cohorts were treated equally often with ICB, but the frequency of achieving a partial response was significantly higher in cohort B $(29 \%)$ than in A $(5 \% ; p=0.018)$ (Table 2). Strikingly, the PR to combined ICB was $5 \%$ vs. $41 \%$ for cohorts A and B, respectively $(p=0.019)$. The median PFS after ICB was 1.9 months (95\% CI 1.3-2.6) vs. 4.2 months (95\% CI 3.0-8.0) for cohorts A and B, respectively $(p<0.001$; Fig. $4 \mathrm{D})$. ICB reinduction was 
A

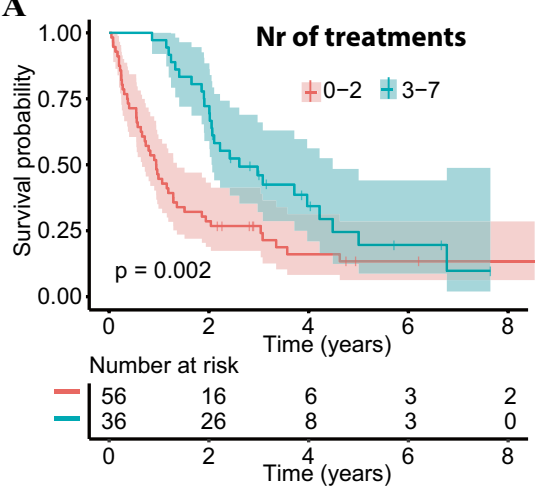

D

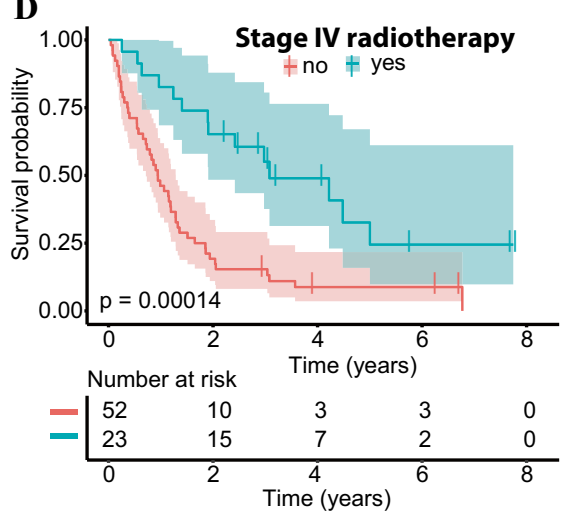

G

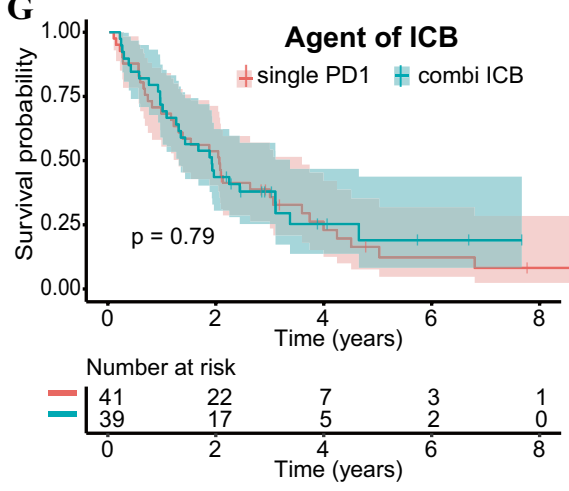

B

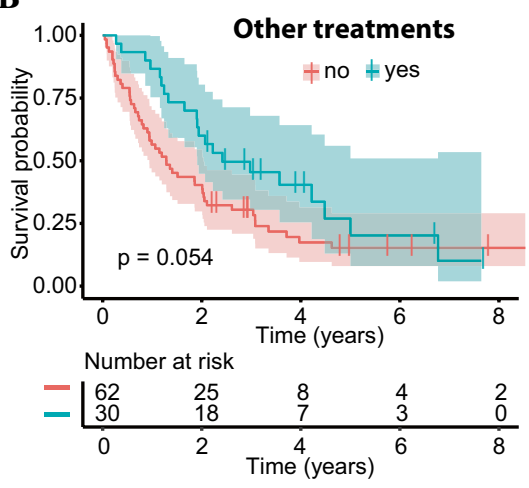

$\mathbf{E}$
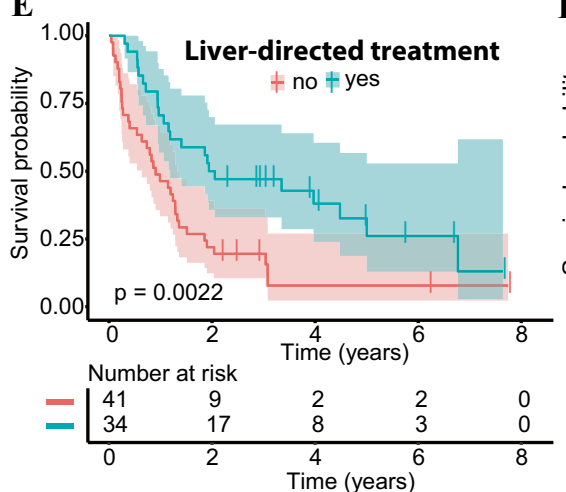

$\mathbf{H}_{1}$
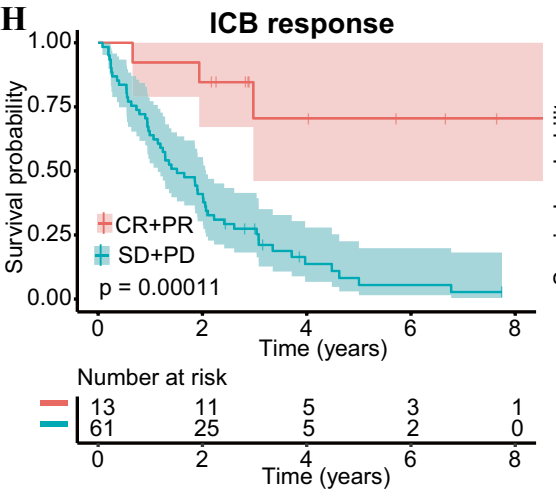

F 1.00 ICB treatment

t no + yes

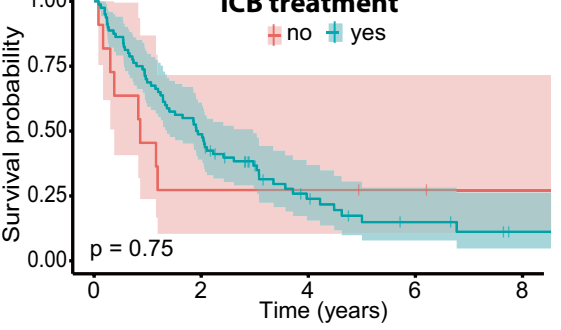

Number at risk

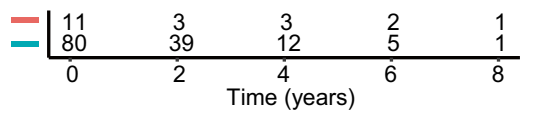

I Reinduction ICB
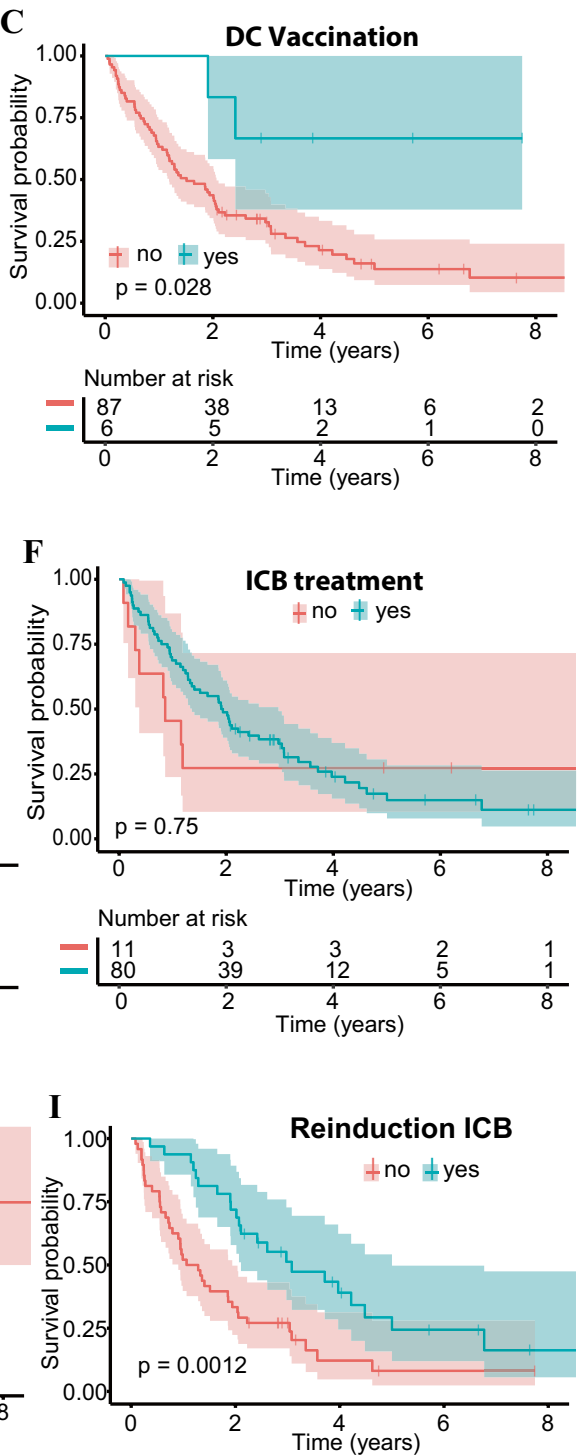

Number at risk

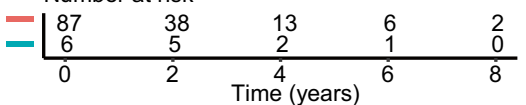

Number at risk

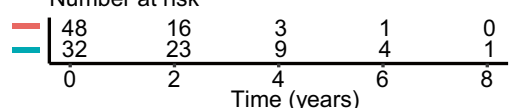

Fig. 3 A Kaplan-Meier estimates for overall survival (OS) according to the number of treatments. The median OS was 11.3 (95\% CI 7.9-16.3) for patients with $\leq 2$ treatment lines vs. 31.3 months $(95 \%$ CI 24.7-53.8) for those with $\geq 3$ treatment lines. B OS according to "other" therapies. The median OS was 15.5 (95\% CI 14.6-24.5) for patients with no "other" therapies vs. 29.0 months (95\% CI 22.9-NR with "other" therapies. C OS according to DC vaccination $(\mathrm{HR} \approx 0.24$, $p=0.04$ ). The median OS was 18.2 months (95\% CI 14.2-24.8) for patients without DC vaccination and not reached with DC vaccination. D OS according to radiation therapy in stage IV $(\mathrm{H} \approx 0.33$, $p<0.001$ ). The median OS was 11.3 (95\% CI 8.6-15.5) for patients without radiation vs. 27.0 months (95\% CI 22.9-NR) for those receiving radiation. $\mathbf{E}$ OS according to liver-directed treatments $(\mathrm{HR} \approx 0.44$, $p=0.003$ ). The median OS was 10.9 (95\% CI 7.6-15.9) for patients without liver-directed treatments vs. 24.0 months (95\% CI 14.2-NR) with liver-directed treatments. F OS according to immune checkpoint inhibitor blockade (ICB). The median OS was 10.3 (95\% CI 4.5-NR) vs. 23.1 months (95\% CI 16.3-35.8). G OS according to different agents of ICB. The median OS was 24.5 (95\% CI 15.4-42.9) for patients with single ICB treatment vs. 22.8 months (95\% CI 15.540.2) with combined ICB. H OS according to the response to ICB. The median OS was not reached for patients with complete or partial response $(\mathrm{CR}+\mathrm{PR})$ vs. 18.2 months $(95 \% \mathrm{CI} 13.8-24.8)$ for those without a response. I OS according to ICB reinduction $(\mathrm{HR} \approx 0.48$, $p=0.004)$. The median OS was 14.1 (95\% CI 10.9-23.3) for patients without ICB reinduction vs. 37.0 months (95\% CI 25.3-60.1) with reinduction. $\mathrm{NR}=$ not reached 

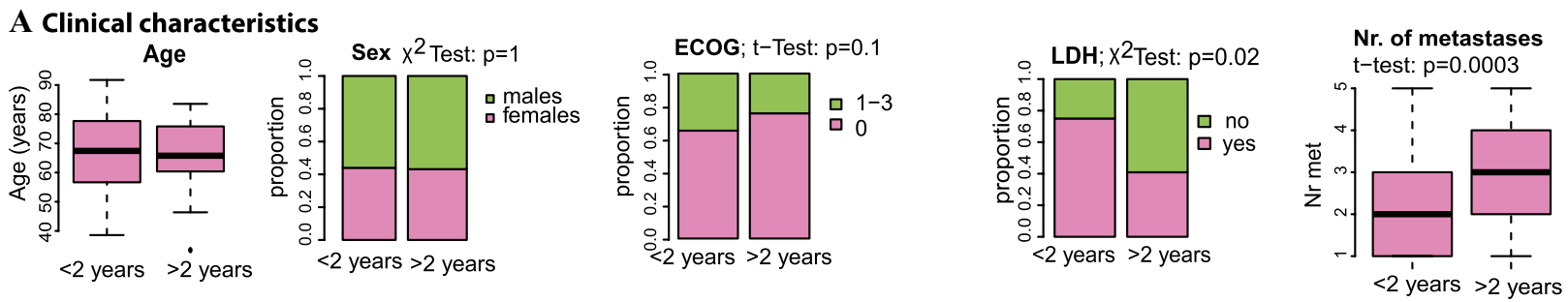

\section{B Metastatic sites}
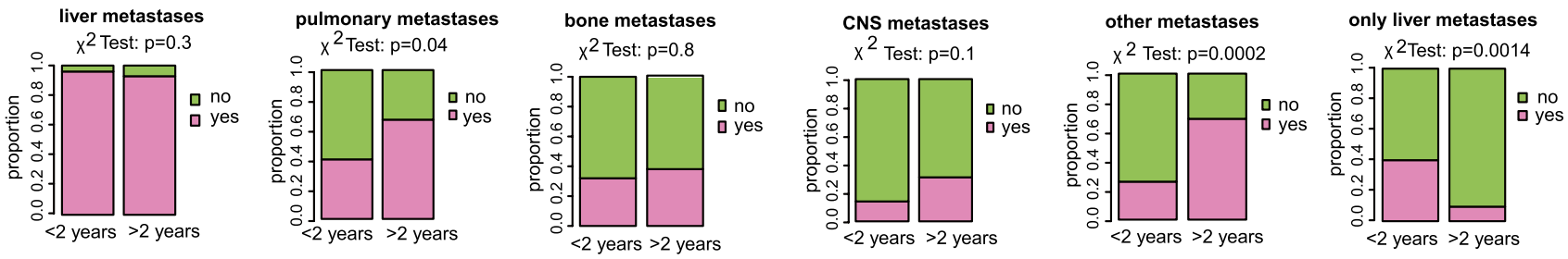

\section{Treatment response}
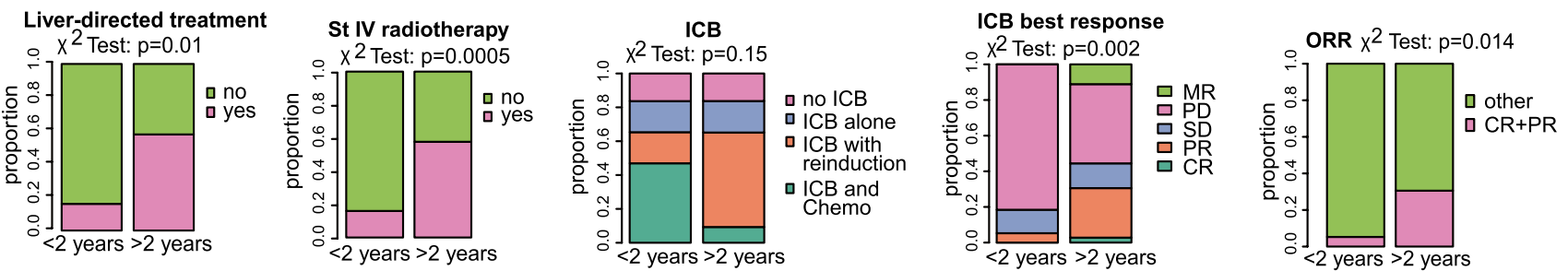

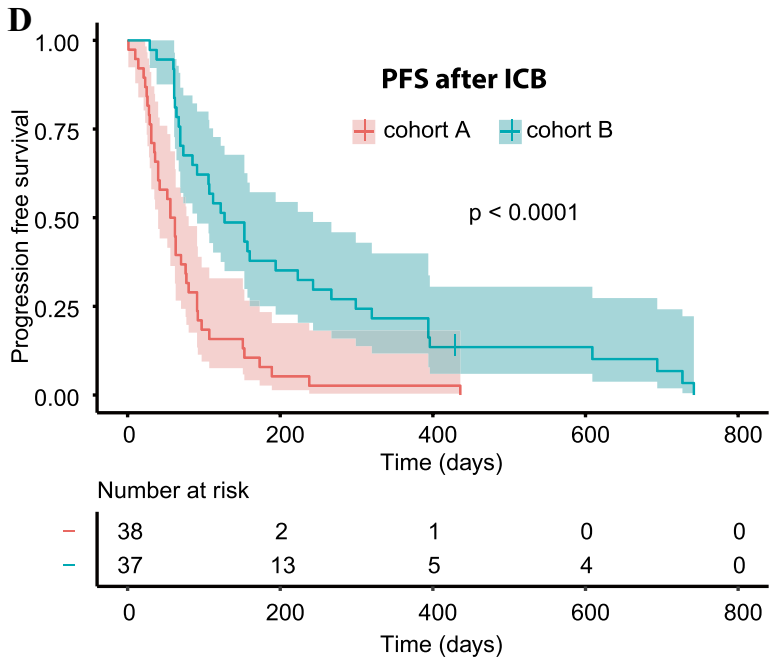

Fig. 4 Investigation of the distribution of prognostic factors in shortterm (cohort A) vs. long-term (cohort B) survivors. A Clinical characteristics; B metastatic sites; C patterns of treatment response. D Kaplan-Meier estimates comparing the cohorts A and B for progression-free survival (PFS) after immune checkpoint inhibitor blockade

more frequent in cohort $\mathrm{B}(p=0.001)$. Also, long-term survivors more commonly underwent radiation therapy $(p<0.001)$ or liver-directed treatments $(p=0.01$; Table 1$)$. The median PFS to the first palliative treatment of any type was 1.9 months (95\% CI 1.9-3.1) vs. 5.0 months (95\% CI 2.7-13.6), respectively ( $p<0.001$; Fig. 4D).

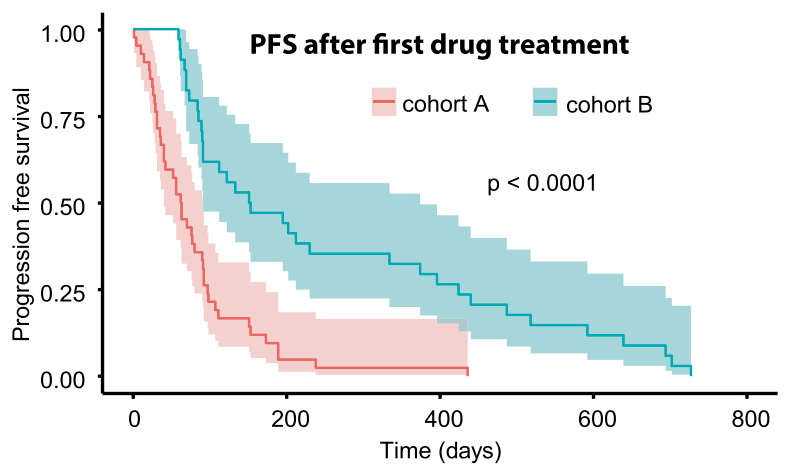

Number at risk

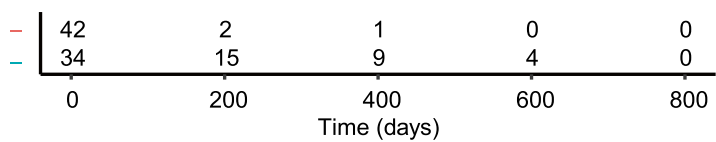

(ICB) and after any first drug treatment in stage IV. The median PFS in stage IV disease after ICB was 1.9 (95\% CI 1.3-2.6) for cohort A vs. 4.2 months (95\% CI 3.0-8.0) for cohort B (left). After the first drug therapy, the median PFS was 2.1 (95\% CI 1.3-3.0) for cohort A vs. 5.0 months (95\% CI 3.0-12.3) for cohort B (right)

\section{Discussion}

Considering that there are no standardized treatment options and only a few high-quality trials for metastatic UM, data from a real-world setting and registries are of particular value for this orphan cancer condition. In this study, we 
followed a two-step strategy to identify clinical parameters that are associated with a favorable outcome. First, we assembled a population from multiple centers within Germany and registry data and performed univariate analyses to explore possible prognostic factors. Second, we further validated these parameters after the population was divided into two cohorts with survival of less and more than 2 years as a proxy for short-term and long-term survival, respectively. Thus, patients with an unknown death status or ongoing treatment within two years after entry into stage IV were excluded. This selection of the population may explain why the OS was relatively high compared to previous studies [10-12]. The median OS for the entire population was 22.3 months which is by far longer than the proposed benchmark of 10.2 months in a recent meta-analysis of 912 patients from clinical trials but comparable to monocentric data other sites [13, 14]. Furthermore, this difference could be explained by a higher proportion of patients treated with ICB. The benchmark study included patients from numerous clinical trials before ICB was available. Accordingly, the proportion of patients receiving immunotherapy was also only $15 \%$, while $34 \%$ were still treated with chemotherapy [14]. In our study, a large proportion received ICB at least once in stage IV disease. The PR rate to single PD1 inhibition and combined ICB were $10 \%$ and $21 \%$, respectively. These results are slightly higher than in two recently published phase II trials $[15,16]$. Piulats et al. achieved a PR rate of $10 \%$ in 52 treatment-naïve patients in the Spanish GEM-1402 trial. A higher PR rate of $15 \%$ was reported by Pelster et al. in 33 patients with any prior treatment for UM. One CR occurred in each of these two prospective studies. Similarly, Pelster et al. reported more favorable OS and PFS values than in the Spanish trial (5.5 vs. 3 months and 19.1 vs. 12.7 months, respectively). These data are in line with our results from a real-world setting and clearly beat the benchmark values [17]. Although the response rates to ICB are by far lower than in cutaneous melanoma, we conclude that the overall prognosis of metastatic UM has been improved significantly by the introduction of ICB.

Our results confirm that a good ECOG performance status and normal serum LDH levels are important prognostic factors in stage IV UM, as was outlined by us and others [11] [18]. Male sex and advanced age were not correlated with survival in contrast to the benchmark data. Interestingly, the number of affected organs was significantly higher in cohort $\mathrm{B}$, while patients with liver metastases only were more commonly observed in cohort A. Also, patients with liver metastases only showed significantly worse survival compared to those with a combination of both hepatic and extrahepatic metastases. This distribution is intriguing and was also observed in the two recent phase II studies. Piulats et al. reported that the OS in patients with exclusive liver metastases was shorter than that in patients with metastases in other locations beyond the liver ( 9.2 vs. 23.5 months) and those with both liver and other metastases (15.5 months) $[15,16]$. Pelster et al. achieved a response in 6 patients of that 5 had both liver and extrahepatic metastases. These results imply that exclusive hepatic metastases are a major unfavorable prognostic factor. The liver exerts a particularly immunosuppressive effect, and the hepatic tumor microenvironment may prevent antigen recognition by tumor-infiltrating lymphocytes, even when treated with ICB [19]. However, if extrahepatic metastases are present, this immunosuppressive effect can be bypassed and the resistance to ICB can be overcome more easily. This hypothesis can help to explain the feeble immune response in UM as $95 \%$ of the metastases spread to the liver which is, in general, the least responsive metastatic site to ICB $[6,20]$. Also, $93 \%$ of the patients included in our study had liver metastases with no significant difference between the cohorts ( $96 \%$ vs $89 \%$ ), whereas the longterm survivors had significantly more frequently pulmonary and "other" metastases. Besides, the number of patients in cohort B with exclusive liver metastases was substantially lower (20 vs. 5 patients). It is noteworthy that the patients with liver metastases only had shorter OS compared to those with several metastatic sites (7.7 vs. 24.8 months) although the overall tumor burden is likely to be higher in the latter group. Consequently, rapidly progressive liver metastases were, counterintuitively, associated with a worse overall survival, while patients with extrahepatic metastasis had a more favorable disease course. We conclude that the presence of multiple affected organ systems with extrahepatic disease is associated with either a more indolent disease course per se or results in better treatment responses. These data support another interesting hypothesis, i.e., that liver metastases cause the elimination of $\mathrm{CD} 8^{+} \mathrm{T}$-cells via immunosuppressive effects of macrophages [21]. This elimination of T-cells may lead to a poor response to ICB in patients with liver metastases only. Besides, liver metastases of UM have a significantly lower PD-L1 expression compared to metastases of cutaneous melanoma, which might also be a reason for the poor response [22-24]. Interestingly, the absence of melanin in liver metastases of UM induces stronger immune responses and is associated with an improved ICB response [25]. However, the hepatic tumor microenvironment might be influenced positively by liver-directed treatments. In our population, patients receiving liver-directed treatments showed significant survival advantages. It remains unclear whether this benefit is truly achieved by liver-directed treatment or whether patients with a more isolated disease are selected for this procedure that per se have a better prognosis. Nonetheless, these interesting signals observed by us and others should be further investigated in future studies.

The limitations of our study are its retrospective design and the exclusion of patients with an unknown death status within 2 years after entry into stage IV that may positively 
bias the survival. Also, the genotype has proven influence on the prognosis but was not considered here due to missing data as no data on the genetic background of the tumors were available.

To date, we have some information on the impact of genetic alterations (1) on development and the pattern of metastasis formation, (2) on the disease course once metastasis has developed, and (3) on the treatment response, in particular to ICB. UM is genetically characterized by chromosomal losses and gains as well as a low mutational burden. Chromosomal alterations like monosomy 3 , disomy 3 , and gain of chromosome $8 \mathrm{q}$ are considered strong prognostic factors. Monosomy 3 is associated with poor disease-free survival (DFS), while tumors with disomy 3 tend to spread rarely and have prolonged DFS. Gain of chromosome $8 \mathrm{q}$ and loss of chromosome 3 are associated with a poor OS [26, 27]. Somatic GNAQ and GNA11 mutations are commonly detected leading to consecutive activation of the MAPK pathway which might contribute to the development of UM [28]. However, survival seems not to be affected by these mutations [29]. Further, hemizygous mutations in the BAP-1 gene were found in monosomy 3 tumors resulting in loss or dysfunctional BAP-1 expression associated with poor DFS and OS [30-32]. Szalai et al. demonstrated a strong association between BAP-1 mutated tumors and a peak of clinical detected metastases 2 years after enucleation [33]. They suggested that this might be an explanation for the observations of the disproved Zimmerman-McLean-Forster effect, who hypothesized that the enucleation causes a spread of tumor cells leading to the peak of metastases $[33,34]$. However, BAP-1 mutated tumors might correlate to locally fast progressive liver metastasis observed in our study population. Moreover, SF3B1 and EIF1AX mutations occur in tumors with disomy 3 and are associated with a more favorable disease course [32, 35, 36]. SF3B1 mutations correlate with a long DFS and with a late peak of metastases after 7 years [30,33]. EIF1AX mutations as well as wild-type BAP-1, SF3B1, and EIF1AX genes are correlated with prolonged survival [30]. Thus, the genotype plays an important role in determining the overall prognosis regardless of treatments. Further, there are signals that a high mutational burden is predictive for response to ICB [37] and a MBD4-related hypermutator phenotype in metastatic uveal melanoma showed an exceptional high sensitivity to anti-PD-1 which is present in up to $2 \%$ of UM patients [38].

Altogether, the prognosis of metastatic UM in this population was better than the recently proposed benchmark and may have improved by the more frequent use of ICB. We demonstrate that a good response to ICB, extrahepatic disease, and liver-directed treatments, were associated with long-term survival.
Supplementary Information The online version contains supplementary material available at https://doi.org/10.1007/s00262-021-03090-4.

Author contributions EATK collected and merged the data into a central database before analysis. AP conducted the statistical analyses. AW designed the figures. EATK and MVH wrote the draft of the manuscript. All other authors were involved in data collection and analysis on-site. All authors were involved in revising the manuscript.

Funding Open Access funding enabled and organized by Projekt DEAL. MVH was supported by the clinician scientist program awarded by the German Society of Dermatology (DDG) and the Arbeitsgemeinschaft Dermatologische Forschung (ADF).

\section{Declarations}

Conflict of interest Carola Berking reports personal fees and nonfinancial support from BMS, personal fees from MSD, personal fees from Novartis, personal fees from Leo Pharma, personal fees from Regeneron, personal fees from Immunocore, personal fees from Sanofi-Genzyme, personal fees from Sanofi-Aventis, personal fees from Almirall-Hermal, personal fees from Roche, personal fees from Pierre Fabre, from Merck, outside the submitted work. Lucie Heinzerling declares research support from Novartis; speakers and advisory board honoraria from Bristol Myers Squibb, CureVac, Merck Sharp \& Dohme, Novartis, Pierre Fabre, Sanofi, and Roche. Beatrice Schell reports receiving speaker's honoraria and/or travel support from Amgen, BMS, MSD, Janssen, Novartis, Roche, Sun Pharma, Abbvie, and Pfizer. Max Schlaak reports receiving honoraria and participation in advisory boards of Bristol Myers Squibb, Novartis, MSD, Roche, Pierre Fabre, Kyowa Kirin, Immunocore, and Sanofi-Genzyme. Max Schlaak received travel accommodation and expenses from Novartis, Pierre Fabre, and Sun Pharma. Patrick Terheyden received speaker's honoraria from BMS, Novartis, MSD, Pierre Fabre, CureVac, and Roche, consultant's honoraria from BMS, Novartis, Pierre Fabre, Merck Serono, Sanofi und Roche and travel support from BMS, Pierre Fabre and Roche. Selma Ugurel declares research support from Bristol Myers Squibb and Merck Serono; speakers and advisory board honoraria from Bristol Myers Squibb, Merck Sharp \& Dohme, Merck Serono, Novartis and Roche, and travel support from Bristol Myers Squibb, and Merck Sharp \& Dohme. Jochen Utikal is on the advisory board or has received honoraria and travel support from Amgen, Bristol Myers Squibb, GSK, LeoPharma, Merck Sharp and Dohme, Novartis, Pierre Fabre, Roche, Sanofi outside the submitted work. Michael Weichenthal reports grants and personal fees from Merck, grants and personal fees from Bristol Myers Squibb, grants and personal fees from Novartis, grants and personal fees from Millennium, personal fees from Kyowa Kirin, personal fees from Takeda, personal fees from Medac, personal fees from Sun Pharma, personal fees from Pierre Fabre, personal fees from Roche, personal fees from Sanofi, outside the submitted work. Fabian Ziller reports personal fees from Bristol Myers Squibb GmbH \& Co KG, Munich, personal fees from MSD SHARP \& DOHME GmbH, Haar, personal fees from Roche Pharma AG, Grenzach-Wyhlen, personal fees from GlaxoSmithKline GmbH \& Co KG, Munich, personal fees from Sanofi-Aventis GmbH, Berlin, personal fees from Novartis Pharma GmbH, Nürnberg, outside the submitted work. All other authors declare no conflict of interest.

Ethical approval This study was approved by the scientific board of the registry and by the institutional review board of the medical faculty of the Munich University Hospital (Approval Number 413-16 UE) and conducted following the principles of the Helsinki Declaration in its current version. 
Open Access This article is licensed under a Creative Commons Attribution 4.0 International License, which permits use, sharing, adaptation, distribution and reproduction in any medium or format, as long as you give appropriate credit to the original author(s) and the source, provide a link to the Creative Commons licence, and indicate if changes were made. The images or other third party material in this article are included in the article's Creative Commons licence, unless indicated otherwise in a credit line to the material. If material is not included in the article's Creative Commons licence and your intended use is not permitted by statutory regulation or exceeds the permitted use, you will need to obtain permission directly from the copyright holder. To view a copy of this licence, visit http://creativecommons.org/licenses/by/4.0/.

\section{References}

1. Aronow ME, Topham AK, Singh AD (2018) Uveal melanoma: 5-year update on incidence, treatment, and survival (SEER 19732013). Ocul Oncol Pathol 4:145-151. https://doi.org/10.1159/ 000480640

2. Mallone S, De Vries E, Guzzo M et al (2012) Descriptive epidemiology of malignant mucosal and uveal melanomas and adnexal skin carcinomas in Europe. Eur J Cancer 48:1167-1175. https:// doi.org/10.1016/j.ejca.2011.10.004

3. Baily C, O'Neill V, Dunne M, Cunningham M, Gullo G, Kennedy S, Walsh PM, Deady S, Horgan N (2019) Uveal melanoma in Ireland. Ocul Oncol Pathol 5:195-204. https://doi.org/10.1159/ 000492391

4. Sayan M, Mamidanna S, Oncel D, Jan I, Vergalasova I, Weiner J, Ohri N, Acikalin B, Chundury A (2020) Clinical management of uveal melanoma: a comprehensive review with a treatment algorithm. Radiat Oncol J 38:162-169. https://doi.org/10.3857/ roj. 2020.00318

5. Fili M, Trocme E, Bergman L et al (2020) Ruthenium-106 versus iodine- 125 plaque brachytherapy of 571 choroidal melanomas with a thickness of $>/=5.5 \mathrm{~mm}$. Br J Ophthalmol 104:26-32. https://doi.org/10.1136/bjophthalmol-2018-313419

6. Collaborative Ocular Melanoma Study G (2001) Assessment of metastatic disease status at death in 435 patients with large choroidal melanoma in the Collaborative Ocular Melanoma Study (COMS): COMS report no. 15. Arch Ophthalmol. 119: 670-6. https://doi.org/10.1001/archopht.119.5.670

7. Kujala E, Makitie T, Kivela T (2003) Very long-term prognosis of patients with malignant uveal melanoma. Invest Ophthalmol Vis Sci 44:4651-4659. https://doi.org/10.1167/iovs.03-0538

8. Franklin C, Livingstone E, Roesch A, Schilling B, Schadendorf D (2017) Immunotherapy in melanoma: recent advances and future directions. Eur J Surg Oncol 43:604-611. https://doi.org/ 10.1016/j.ejso.2016.07.145

9. Rantala ES, Hernberg M, Kivela TT (2019) Overall survival after treatment for metastatic uveal melanoma: a systematic review and meta-analysis. Melanoma Res 29:561-568. https://doi.org/10. 1097/CMR.0000000000000575

10. Heppt MV, Amaral T, Kahler KC et al (2019) Combined immune checkpoint blockade for metastatic uveal melanoma: a retrospective, multi-center study. J Immunother Cancer 7:299. https://doi. org/10.1186/s40425-019-0800-0

11. Heppt MV, Heinzerling L, Kahler KC et al (2017) Prognostic factors and outcomes in metastatic uveal melanoma treated with programmed cell death-1 or combined PD-1/cytotoxic T-lymphocyte antigen-4 inhibition. Eur J Cancer 82:56-65. https://doi.org/10. 1016/j.ejca.2017.05.038

12. Kuk D, Shoushtari AN, Barker CA et al (2016) Prognosis of mucosal, uveal, acral, nonacral cutaneous, and unknown primary melanoma from the time of first metastasis. Oncologist 21:848854. https://doi.org/10.1634/theoncologist.2015-0522

13. Kirchberger MC, Moreira A, Erdmann M, Schuler G, Heinzerling L (2018) Real world experience in low-dose ipilimumab in combination with PD-1 blockade in advanced melanoma patients. Oncotarget 9:28903-28909. https://doi.org/10.18632/oncotarget. 25627

14. Khoja L, Atenafu EG, Suciu S et al (2019) Meta-analysis in metastatic uveal melanoma to determine progression free and overall survival benchmarks: an international rare cancers initiative (IRCI) ocular melanoma study. Ann Oncol 30:1370-1380. https:// doi.org/10.1093/annonc/mdz176

15. Piulats JM, Espinosa E, de la Cruz ML et al (2021) Nivolumab plus ipilimumab for treatment-naive metastatic uveal melanoma: an open-label, multicenter, phase II trial by the Spanish multidisciplinary melanoma group (GEM-1402). J Clin Oncol. https://doi. org/10.1200/JCO.20.00550

16. Pelster MS, Gruschkus SK, Bassett R et al (2020) Nivolumab and ipilimumab in metastatic uveal melanoma: results from a singlearm phase II study. J Clin Oncol. https://doi.org/10.1200/JCO.20. 00605

17. Khan S, Carvajal RD (2021) Dual immunological checkpoint blockade for uveal melanoma. J Clin Oncol. https://doi.org/10. 1200/JCO.20.03274

18. Nicholas MN, Khoja L, Atenafu EG, Hogg D, Quirt I, Butler M, Joshua AM (2018) Prognostic factors for first-line therapy and overall survival of metastatic uveal melanoma: the princess margaret cancer centre experience. Melanoma Res 28:571-577. https://doi.org/10.1097/CMR.0000000000000468

19. Lindblad KE, Lujambio A (2021) Liver metastases inhibit immunotherapy efficacy. Nat Med 27:25-27. https://doi.org/10.1038/ s41591-020-01190-9

20. Pires da Silva I, Lo S, Quek C, Gonzalez M, Carlino MS, Long GV, Menzies AM (2020) Site-specific response patterns, pseudoprogression, and acquired resistance in patients with melanoma treated with ipilimumab combined with anti-PD-1 therapy. Cancer 126:86-97. https://doi.org/10.1002/cncr.32522

21. Somasundaram R, Connelly T, Choi R et al (2021) Tumorinfiltrating mast cells are associated with resistance to antiPD-1 therapy. Nat Commun 12:346. https://doi.org/10.1038/ s41467-020-20600-7

22. Hoefsmit EP, Rozeman EA, Van TM et al (2020) Comprehensive analysis of cutaneous and uveal melanoma liver metastases. J Immunother Cancer. https://doi.org/10.1136/jitc-2020-001501

23. Zou W, Wolchok JD, Chen L (2016) PD-L1 (B7-H1) and PD-1 pathway blockade for cancer therapy: mechanisms, response biomarkers, and combinations. Sci Transl Med. https://doi.org/10. 1126/scitranslmed.aad7118

24. Wessely A, Steeb T, Erdmann M, Heinzerling L, Vera J, Schlaak M, Berking C, Heppt MV (2020) The role of immune checkpoint blockade in uveal melanoma. Int J Mol Sci. https://doi.org/10. 3390/ijms21030879

25. Rothermel LD, Sabesan AC, Stephens DJ et al (2016) Identification of an immunogenic subset of metastatic uveal melanoma. Clin Cancer Res 22:2237-2249. https://doi.org/10.1158/10780432.CCR-15-2294

26. Prescher G, Bornfeld N, Hirche H, Horsthemke B, Jockel KH, Becher R (1996) Prognostic implications of monosomy 3 in uveal melanoma. Lancet 347:1222-1225. https://doi.org/10.1016/s01406736(96)90736-9

27. Sisley K, Rennie IG, Parsons MA, Jacques R, Hammond DW, Bell SM, Potter AM, Rees RC (1997) Abnormalities of chromosomes 3 and 8 in posterior uveal melanoma correlate with prognosis. Genes Chromosomes Cancer 19:22-28. https://doi.org/10.1002/ (sici)1098-2264(199705)19:1\%3c22::aid-gcc4\%3e3.0.co;2-2 
28. Van Raamsdonk CD, Griewank KG, Crosby MB et al (2010) Mutations in GNA11 in uveal melanoma. N Engl J Med 363:2191-2199. https://doi.org/10.1056/NEJMoa1000584

29. Koopmans AE, Vaarwater J, Paridaens D, Naus NC, Kilic E, de Klein A, Rotterdam Ocular Melanoma Study g (2013) Patient survival in uveal melanoma is not affected by oncogenic mutations in GNAQ and GNA11. Br J Cancer. 109: 493-6. https://doi.org/ 10.1038/bjc.2013.299

30. Yavuzyigitoglu S, Koopmans AE, Verdijk RM et al (2016) Uveal melanomas with SF3B1 mutations: a distinct subclass associated with late-onset metastases. Ophthalmology 123:1118-1128. https://doi.org/10.1016/j.ophtha.2016.01.023

31. Kalirai H, Dodson A, Faqir S, Damato BE, Coupland SE (2014) Lack of BAP1 protein expression in uveal melanoma is associated with increased metastatic risk and has utility in routine prognostic testing. Br J Cancer 111:1373-1380. https://doi.org/10.1038/bjc. 2014.417

32. Robertson AG, Shih J, Yau C et al (2017) Integrative analysis identifies four molecular and clinical subsets in uveal melanoma. Cancer Cell 32(204-20):e15. https://doi.org/10.1016/j.ccell.2017. 07.003

33. Szalai E, Jiang Y, van Poppelen NM, Jager MJ, de Klein A, Kilic E, Grossniklaus HE (2018) Association of uveal melanoma metastatic rate with stochastic mutation rate and type of mutation. JAMA Ophthalmol 136:1115-1120. https://doi.org/10.1001/ jamaophthalmol.2018.2986

34. Singh AD, Rennie IG, Kivela T, Seregard S, Grossniklaus H (2004) The Zimmerman-Mclean-Foster hypothesis: 25 years later.

\section{Authors and Affiliations}

Elias A. T. Koch ${ }^{1,2} \cdot$ Anne Petzold $^{1,2} \cdot$ Anja Wessely $^{1,2} \cdot$ Edgar Dippel $^{3} \cdot$ Michael Erdmann $^{1,2} \cdot$ Lucie Heinzerling $^{1,4}$. Bettina Hohberger ${ }^{5}$. Harald Knorr ${ }^{5}$. Ulrike Leiter ${ }^{6}$. Friedegund Meier ${ }^{7}$. Peter Mohr ${ }^{8}$. Farnaz Rahimi ${ }^{4}$. Beatrice Schell ${ }^{9} \cdot$ Max Schlaak $^{4,10}$. Patrick Terheyden ${ }^{11}$. Beatrice Schuler-Thurner ${ }^{1,2}$. Selma Ugurel ${ }^{12}$. Jochen Utikal ${ }^{13,14}$. Julio Vera ${ }^{1,2} \cdot$ Michael Weichenthal $^{15} \cdot$ Fabian Ziller $^{16} \cdot$ Carola Berking $^{1,2} \cdot$ Markus V. Heppt $^{1,2}$ (I)

1 Department of Dermatology, Universitätsklinikum Erlangen, Friedrich-Alexander-University Erlangen-Nürnberg (FAU), Erlangen, Germany

2 Deutsches Zentrum Immuntherapie (DZI), Comprehensive Cancer Center Erlangen-European Metropolitan Area of Nuremberg (CCC ER-EMN), Erlangen, Germany

3 Department of Dermatology, Ludwigshafen Medical Center, Ludwigshafen, Germany

4 Department of Dermatology and Allergy, Munich University Hospital (LMU), Munich, Germany

5 Department of Ophthalmology, Universitätsklinikum Erlangen, Friedrich-Alexander-University Erlangen-Nürnberg (FAU), Erlangen, Germany

6 Department of Dermatology, Center for Dermatooncology, University Hospital Tübingen, Tübingen, Germany

7 Department of Dermatology, Skin Cancer Center, Medical Faculty, University Hospital Carl Gustav Carus, TU Dresden, Dresden, Germany

8 Department of Dermatology, Elbe Klinikum Buxtehude, Buxtehude, Germany
Br J Ophthalmol 88:962-967. https://doi.org/10.1136/bjo.2003. 029058

35. Ewens KG, Kanetsky PA, Richards-Yutz J, Purrazzella J, Shields CL, Ganguly T, Ganguly A (2014) Chromosome 3 status combined with BAP1 and EIF1AX mutation profiles are associated with metastasis in uveal melanoma. Invest Ophthalmol Vis Sci 55:5160-5167. https://doi.org/10.1167/iovs.14-14550

36. Furney SJ, Pedersen M, Gentien D et al (2013) SF3B1 mutations are associated with alternative splicing in uveal melanoma. Cancer Discov 3:1122-1129. https://doi.org/10.1158/2159-8290. CD-13-0330

37. Rizvi NA, Hellmann MD, Snyder A et al (2015) Cancer immunology. Mutational landscape determines sensitivity to PD-1 blockade in non-small cell lung cancer. Science 348:124-128. https:// doi.org/10.1126/science.aaa1348

38. Rodrigues M, Mobuchon L, Houy A et al (2018) Outlier response to anti-PD1 in uveal melanoma reveals germline MBD4 mutations in hypermutated tumors. Nat Commun 9:1866. https://doi.org/10. 1038/s41467-018-04322-5

Publisher's Note Springer Nature remains neutral with regard to jurisdictional claims in published maps and institutional affiliations.
9 Department of Dermatology, SRH Wald-Klinikum Gera, Gera, Germany

10 Department of Dermatology, Venerology and Allergology, Charité - Universitätsmedizin Berlin, Corporate Member of Freie Universität Berlin, Humboldt-Universität, Berlin, Germany

11 Department of Dermatology, Allergology and Venereology, University Medical Center of Schleswig-Holstein, Campus, Lübeck, Germany

12 Department of Dermatology, University Hospital Essen, University Duisburg-Essen, Duisurg, Germany

13 Skin Cancer Unit, German Cancer Research Center (DKFZ), Heidelberg, Germany

14 Department of Dermatology, Venereology and Allergology, University Medical Center Mannheim, Ruprecht-Karl University of Heidelberg, Mannheim, Germany

15 Department of Dermatology, University Hospital Schleswig-Holstein, Campus Kiel, Kiel, Germany

16 Department of Dermatology, DRK Krankenhaus Rabenstein, Chemnitz, Germany 